\title{
Random Filters for Compressive Sampling
}

\author{
Joel A. Tropp \\ Department of Mathematics \\ The University of Michigan at Ann Arbor \\ 530 Church St., Ann Arbor, MI 48109-1043 \\ E-mail: jtropp@umich.edu
}

\begin{abstract}
This paper discusses random filtering, a recently proposed method for directly acquiring a compressed version of a digital signal. The technique is based on convolution of the signal with a fixed FIR filter having random taps, followed by downsampling. Experiments show that random filtering is effective at acquiring sparse and compressible signals. This process has the potential for implementation in analog hardware, and so it may have a role to play in new types of analog/digital converters.
\end{abstract}

\section{INTRODUCTION}

Many types of signals have additional structure that makes them compressible. In other words, the amount of information necessary to represent or approximate the signal is substantially smaller than the length of the signal. Nevertheless, many techniques for signal acquisition collect a complete description of the signal, just to throw away the redundant data. For example, audio signals are typically sampled at the Nyquist rate before lossy compression algorithms are applied to reduce their size. While the sample-and-compress approach is attractive when the Nyquist rate is $44 \mathrm{KHz}$ (as with CD-quality audio), it becomes increasingly distasteful as the sampling rate increases. In modern applications, it may be necessary to acquire precise information about signals with Nyquist rates into and above the gigahertz range. This task is hopeless for current analog/digital converters.

The pressure to develop novel technologies for signal acquisition has led researchers to look beyond Shannon-Nyquist sampling. In this paper, we discuss random filtering, a recently proposed technique for directly acquiring a compressed version of a digital signal [1]. We believe that this method may also be applicable to analog signals, and so it may have a role to play in next-generation $\mathrm{A} / \mathrm{D}$ converters.

\section{Compressive Signal Acquisition}

Let us consider a simple (but representative) situation. We will work in the vector space $\mathbb{R}^{d}$, equipped with the usual Euclidean norm $\|\cdot\|_{2}$. Define the class of $m$-sparse signals

$$
B_{0}(m)=\left\{\boldsymbol{s} \in \mathbb{R}^{d}: \# \operatorname{supp}(\boldsymbol{s}) \leq m\right\} .
$$

It is clear that each of these signals can be represented completely using $2 m$ real numbers. When $m$ is substantially smaller than $d$, therefore, it is wasteful to write an $m$-sparse signal in the standard basis. In particular, it seems lavish to take $d$ samples of a sparse signal to identify the $m$ nonzero components.
The theoretical goal of compressive signal acquisition (CSA), aka compressed sensing, is to develop a linear measurement operator $\Phi: \mathbb{R}^{d} \rightarrow \mathbb{R}^{n}$ and a (nonlinear) reconstruction algorithm $\mathscr{A}: \mathbb{R}^{n} \rightarrow \mathbb{R}^{d}$ for which

1) the number $n$ of measurements is comparable with the sparsity level $m$,

2) the measurement process does not discard information, i.e., $B_{0}(m) \cap \operatorname{ker} \boldsymbol{\Phi}=\{\mathbf{0}\}$, and

3) the reconstruction algorithm is stable, i.e.,

$$
\|\mathscr{A}(\boldsymbol{\Phi} \boldsymbol{s}+\boldsymbol{\nu})-\boldsymbol{s}\|_{2} \leq \mathrm{C}\|\boldsymbol{\nu}\|_{2} .
$$

In the sequel, we will discuss more practical aspects of measurement and reconstruction.

Indeed, it is possible to design measurement processes and reconstruction algorithms that satisfy all these requirements. For example, we can choose the measurement map $\Phi$ to be a $d \times n$ Gaussian matrix with $n=O(m \log d)$. In this case, several different algorithms can be used to recover sparse signals. A partial list of references for these results includes [2], [3], [4], [5].

\section{RANDOM FILTERS}

When viewed as candidates for next-generation A/D converters, the current collection of techniques for CSA do not look promising. The major shortcoming is that these approaches use measurement processes that seem incompatible with analog hardware. Indeed, analog hardware can reliably perform only a limited repertoire of operations: (i) modulation, (ii) filtering, and (iii) sampling. It is natural, therefore, to search for a new type of measurement process that can be built using these simple blocks.

There are several other problems with current approaches to CSA that we also wish to circumvent: (i) the methods are only designed for finite-length signals, (ii) the measurement process is not causal, (iii) the reconstruction algorithms require too much time and space.

To address some of these difficulties, the paper [1] proposes a new method for compressive acquisition of digital signals. The idea is that we can measure a signal $s$ by convolving it with an FIR filter $\boldsymbol{h}$ with random taps and then downsampling the result to obtain a compressed representation $y$. Figure 1 displays a block diagram of this filtering process. Various nonlinear reconstruction algorithms are possible. For a summary of the potential advantages of this approach, we quote [1]: 


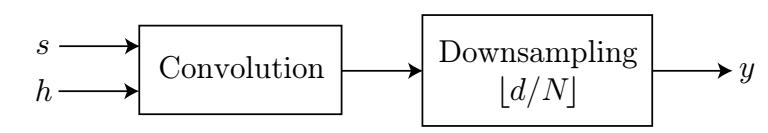

(a)

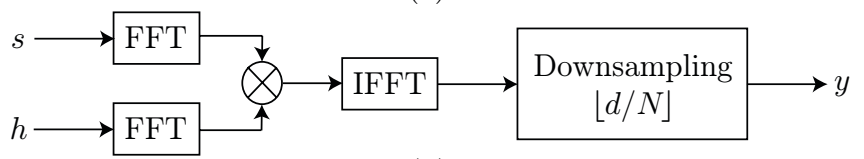

(b)

Fig. 1. Block diagrams for signal acquisition through random filtering: (a) using convolution; (b) using FFT/IFFT. The FIR filter $\boldsymbol{h}$ has random taps, which must be known in order to recover the signal $\boldsymbol{s}$ from the compressed data $\boldsymbol{y}$.

At first glance, one might think this method would convert a signal into garbage. In fact, the random filter is generic enough to summarize many types of compressible signals. At the same time, the random filter has enough structure to accelerate measurement and reconstruction algorithms. Our method has several benefits:

- measurements are time-invariant and nonadaptive;

- measurement operator is stored and applied efficiently;

- we can trade longer filters for fewer measurements;

- it is easily implementable in software or hardware; and

- it generalizes to streaming or continuous-time signals.

The original paper [1] discusses implementation issues connected with random filters, and it describes a reconstruction algorithm based on Orthogonal Matching Pursuit (OMP). We will not repeat this material here. The remainder of the paper describes the numerical evidence about the performance of random filters for CSA. We conclude with a discussion of the challenges that arise in the mathematical analysis.

\section{NUMERICAL EXPERIMENTS}

Extensive numerical experiments indicate that random filters do provide sufficient information to reconstruct sparse signals. In this section, we describe an experiment performed in [1]. We fix the signal length $d=128$, and the sparsity $m=10$. For each number $n$ of measurements and filter length $B$, we do the following. First, draw a random filter of length $B$ with $\mathcal{N}(0,1)$ taps. For each of 1000 trials, we generate a signal $s$ whose $m$ nonzero entries are $\mathcal{N}(0,1)$. We take $n$ measurements and use an OMP-based algorithm to reconstruct the signal. If the results match to machine precision, then we record a success. The reconstruction probability is the fraction of the 1000 trials that result in success. As a control, we perform the same experiment using a fully random matrix $\boldsymbol{\Phi}$ with i.i.d. $\mathcal{N}(0,1)$ entries. Figure 2 displays the results. Note in particular that the two longest filters $(B=64,128)$ succeed almost as well
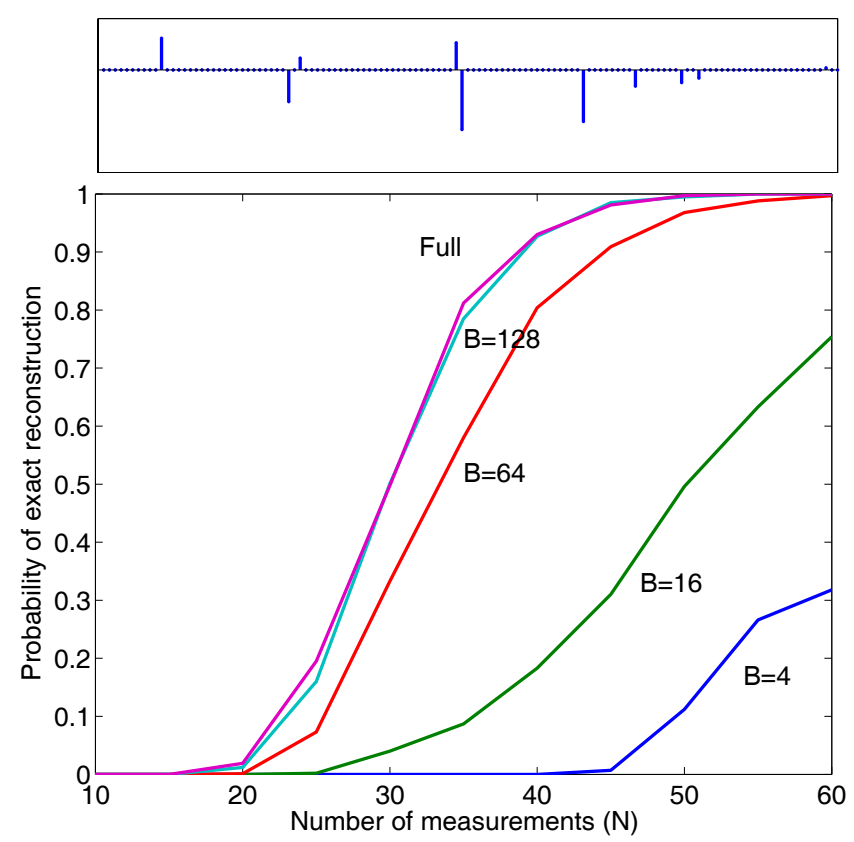

Fig. 2. Probability of exact reconstruction versus the number of measurements $n$ for four filter lengths $B$. Signal length $d=128$. A typical signal appears at top.

as the Gaussian matrix, even though they have fewer degrees of freedom.

\section{Mathematical Analysis of Random Filters}

At present, there are no rigorous mathematical results on the performance of random filters. We are attempting to calculate the restricted isometry constants [6] of a random filter using methods from the study of empirical processes. This calculation would demonstrate that the random filtering process captures all sparse and compressible signals from certain classes. Although it does not seem very hard to develop suppression results (the upper singular value estimates), the corresponding lower estimates remain out of our reach.

\section{ACKNOWLEDGMENTS}

I wish to thank Mike Wakin, Marco Duarte, Dror Baron, and Rich Baraniuk for permission to discuss our joint work.

\section{REFERENCES}

[1] J. A. Tropp, M. B. Wakin, M. F. Duarte, D. Baron, and R. G. Baraniuk, "Random filters for compressive sampling and reconstruction," in Proc. ICASSP 2006, Toulouse, May 2006, to appear.

[2] D. L. Donoho, "Compressed sensing," Oct. 2004, unpublished manuscript

[3] E. J. Candès and T. Tao, "Near optimal signal recovery from random projections: Universal encoding strategies?" Nov. 2004, submitted for publication.

[4] M. Rudelson and R. Veshynin, "Geometric approach to error correcting codes and reconstruction of signals," Feb. 2005, available from arXiv:math.MG/0502299. [Online]. Available: arXiv:math.MG/0502299

[5] J. A. Tropp and A. C. Gilbert, "Signal recovery from partial information via Orthogonal Matching Pursuit," April 2005, submitted to IEEE Trans. Inform. Theory.

[6] E. J. Candès and T. Tao, "Decoding by linear programming," IEEE Trans. Inform. Theory, vol. 51, no. 12, pp. 4203-4215, Dec. 2005. 